\title{
Broadband Load Torque Estimation in Mechatronic Powertrains using Nonlinear Kalman Filtering
}

\author{
Bart Forrier, Frank Naets and Wim Desmet
}

\begin{abstract}
An important bottleneck in the design, operation and exploitation of mechatronic powertrains is the lack of accurate knowledge of broadband external loading. This is caused by the intrusive nature of regular torque measurements. This paper proposes a novel non-intrusive approach to obtain torsional load information on mechatronic powertrains. Online coupled state/input estimation is performed through an augmented nonlinear Kalman filter. This estimation approach exploits general lumped parameter physics-based models in order to create a widely applicable framework. This work considers both extended (EKF) and unscented Kalman filtering (UKF) approaches. Contrary to previous works, no considerable difference in accuracy is obtained from experiments, with a considerably lower computational load for the EKF. This work reveals the benefits of including rotational acceleration measurements from a theoretical perspective, which is demonstrated through experimental validation. This drastically increases the broadband accuracy. The result of this work is an accurate and non-invasive virtual torque sensor with a sufficiently broad bandwidth for use in condition monitoring, control and future design optimization.
\end{abstract}

Index Terms-Input estimation, Kalman filter, mechatronic, nonlinear, observability, torque estimation.

\section{INTRODUCTION}

$\mathbf{T}$ ORSIONAL loading is of paramount importance to operational powertrain performance in terms of noise, vibration and harshness $(\mathrm{NVH})$, structural reliability and efficiency. The difficulty in quantifying transmitted torques is therefore in sharp contrast to their relevance in design and control. This is particularly true for mechatronic powertrains, as efficient and quiet, yet high-dynamic operation is key in industrial and commercial applications.

Torque sensors or flanges instrumented with strain gauges allow a direct measurement of torques at a given location, assuming that the sensor can be practically installed. This is rarely possible in operational systems, limiting the use to inlab situations. Even there, the high level of intrusivity requires special care to not influence the measurement as a result of sensor installation.

Manuscript received November 03, 2016; revised March 8, 2017; Accepted July 24, 2017. This research was supported in part by the Research Fund KU Leuven. It was also supported partially by Flanders Make, the Strategic Research Centre for the Manufacturing Industry. The research of $F$. Naets is funded by a grant from the Research Foundation-Flanders (FWO).

B. Forrier, F. Naets and W. Desmet are with the Department of Mechanical Engineering, KU Leuven, Leuven, Belgium and they are members of Flanders Make (e-mail: bart.forrier@kuleuven.be).
Indirect torque measurement is therefore attempted by means of a relation between the torque and other quantities that are more practical to measure. Specific approaches have been developed for wind turbine condition monitoring [1], [2] and gearbox fault detection [3]-[6]. However, the methods proposed in [1]- [6] are very limited in applicability, as they assume the torques of interest to be the result of internal system behavior and they cannot handle unknown external loads.

The treatment of unknown external torques leads to an inverse problem and poses specific challenges towards higherfrequency loading. The loss of high-frequency torque information when using coupled state/input observer techniques has previously been attributed to damping [7] or absorption due to induction machine rotor inertia [6]. The filtering effect of the mechanical behavior limits the useful bandwidth of any load torque estimation based only on the measurements of electrical quantities at the motor terminals. In order to mitigate this issue, the above literature has focused on specific frequency bands and/or harmonics of the transmitted torques. This can be used for diagnostics of certain driveline effects. However, it does not provide a more comprehensive view on the system behavior as often required for design and control.

In this work, we propose a general approach to merge data from the electrical and mechanical domains in a model-based coupled state/input estimator [8] for mechatronic powertrain torque identification. We employ a general purpose lumpedparameter torsional driveline model including electrical and mechanical dynamics. The electrical part is required for an accurate identification in the low-frequency range.

For linear systems, the problem of coupled state/input estimation using Kalman filter (KF) techniques [9], [10] has been addressed from a theoretical viewpoint [11], [12]. As mechatronic powertrains typically feature prominent nonlinear effects, they require specific treatment. For such nonlinear systems, the unscented Kalman filter (UKF) is often considered more suited than the extended Kalman filter (EKF). This has been concluded in general [13]-[15] and with regard to induction machine (IM) applications [16]. However, we show that for the practical application considered here, the results of the UKF and EKF are practically indistinguishable as a result of the small time-integration step size. For our particular implementation the EKF also computes faster than the UKF.

By feeding a combination of electrical and mechanical data into the mechatronic powertrain model, this work extends the useful load torque estimation bandwidth significantly. 
To achieve this, it relates available model and measurement equations to estimation results. This is done by using readily applicable criteria [17], [18] derived from general nonlinear observability theory [19]. Such a nonlinear observability analysis has already been used in mechatronics to assess the quality of speed and rotor flux estimation assuming constant speed [20] or varying speed operation [21]. The presented work assumes availability of a shaft angle measurement, which is often avoided in research related to IM control. This angle measurement is shown to not be necessary for observability, but is easily implemented in mechatronic applications.

The presented approach explicitly considers the load torque as an unknown input to the model and shows how this affects the overall observability assessment. The results of this observability analysis yields insight in sensor requirements for the online estimation. As the observability provides minimal requirements with respect to necessary sensors, it provides little information on the actual performance of the estimator. For a further assessment we also present a bandwidth analysis of the filter for different sensor sets. This analysis shows how the rotational acceleration measurements drastically increase the achievable bandwidth. Finally, the presented approach is validated experimentally on a mechatronic powertrain test setup [22]. To the authors' knowledge, this is the first experimental validation of a virtual load torque sensor.

\section{Mechatronic Powertrain Model}

The lumped-parameter mechatronic powertrain model is developed in Amesim [23], a commercial software package based on the bond graph theory [24]. The mechatronic powertrain model consists of two main submodels: the electric AC induction machine and the mechanical transmission. These two models and their coupling are discussed in the following three sub-sections.

\section{A. Electrical AC induction machine model}

The two-pole AC induction machine is modeled according to the $\Gamma$-model proposed by Slemon [25]. This 2-phase equivalent model is described by two stator and rotor voltage equations.

$$
\begin{aligned}
\frac{d \mathbf{i}_{\mathbf{s}}}{d t} & =\frac{1}{l_{\sigma s}}\left[\mathbf{u}_{\mathbf{s}}-\left(r_{s}+\frac{L_{m}}{\tau_{r}}\right) \mathbf{i}_{\mathbf{s}}-j \omega \boldsymbol{\Psi}_{\mathbf{r}}^{\prime}\right] \\
\frac{d \boldsymbol{\Psi}_{\mathbf{r}}^{\prime}}{d t} & =-\frac{1}{\tau_{r}} \boldsymbol{\Psi}_{\mathbf{r}}^{\prime}+\frac{L_{m}}{\tau_{r}} \mathbf{i}_{\mathbf{s}}+j \omega \boldsymbol{\Psi}_{\mathbf{r}}^{\prime}
\end{aligned}
$$

In (1)-(2), $\mathbf{u}_{\mathbf{s}}=u_{s \alpha}+j u_{s \beta}$ and $\mathbf{i}_{\mathbf{s}}=i_{s \alpha}+j i_{s \beta}$ are the stator voltage and current phasors, respectively. $\Psi_{r}^{\prime}$ is the part of the stator-referred airgap flux due to current flows in the rotor cage and $\omega$ denotes the electrical rotor speed. The variables of this electrical state space model are grouped in the state vector $\mathbf{x}_{e l}$ :

$$
\mathbf{x}_{e l} \triangleq\left[i_{s \alpha}, i_{s \beta}, \Psi_{r \alpha}^{\prime}, \Psi_{r \beta}^{\prime}\right]^{T} .
$$

The model parameters are the stator resistance $r_{s}$ and leakage inductance $l_{\sigma s}$, the equivalent magnetizing inductance $L_{m}$ and the rotor time constant $\tau_{r}$. Unlike the T-model [25],
[26], this $\Gamma$-model does not describe the actual rotor current or flux phasor, but an equivalent quantity yielding the same inputoutput behavior at the stator. Hence a rotor leakage inductance parameter is not required and the $\Gamma$-model does not suffer from parameter redundancy. As pointed out in [20], all four parameters of the $\Gamma$-model describing a real induction machine can be identified from experimental I/O data.

The coupling with the mechanical model is established through the motor speed ( $\omega_{M}=\omega$ for a two-pole machine) and the electromagnetic torque equation:

$$
T_{e m}^{*}=\Psi_{\mathbf{r}}^{\prime} \times \mathbf{i}_{\mathbf{s}} .
$$

This model was experimentally validated by applying four different reference profiles for the motor speed, while requesting a constant electromagnetic torque from a second IM acting as load. However, this validation indicated a relatively low accuracy of this model for the predicted electromagnetic torque ${ }^{1}$. The authors therefore propose the use of an additional linear transformation superposed on this model, through an additional gain $\kappa$ and offset $\gamma$ :

$$
T_{e m}=\kappa T_{e m}^{*}+\gamma .
$$

The use of this linear transformation (5) to obtain $T_{e m}$ is motivated by the strong correlation between $\hat{T}_{e m}^{*}$ and the resulting estimation error. The additional parameters $\kappa$ and $\gamma$ can obtained through a linear curve-fit on the error between the predicted and measured torques, leading to $\kappa=0.79$ and $\gamma=-0.26 \mathrm{Nm}$ for the the considered machine. For the different speed profiles considered, the variation on these values is less than $2 \%$ for $\kappa$ and $0.1 \mathrm{Nm}$ for $\gamma$, such that the assumption of a one-time calibration seems valid.

The 2-phase equivalent IM model (1)-(4) incorporating these corrections (5) is related to the known terminal voltage inputs $u_{s, a b c}$ and measured currents $i_{s, a b c}$ by Clarke's respective forward and inverse reference frame transformation. This motor model is used in the Amesim system level model.

\section{B. Mechanical cardan shaft transmission model}

Fig. 1 illustrates the lumped parameter model that represents the torsional behavior of the mechanical driveline. This model lumps all flexibility at the location of the intermediate shaft, parameterized by a stiffness $k$ and a damping $c$. Its inertia is much lower than that of the component at motor $\left(J_{M}\right)$ and at load $\left(J_{L}\right)$ side and can be ignored when considering the system-level behavior. The intermediate shaft, referred to by the superscript $\cdot^{*}$, therefore exerts the same torque $T^{*}$ on both cardan joints:

$$
T^{*}=k\left(\theta_{M}^{*}-\theta_{L}^{*}\right)+c\left(\omega_{M}^{*}-\omega_{L}^{*}\right),
$$

where $\omega_{M \mid L}^{*}$ and $\theta_{M \mid L}^{*}$ denote the rotational speeds and angles at the shaft's end yokes, respectively. The mechanical state variables $\mathbf{x}_{m}$ are:

$$
\mathbf{x}_{m} \triangleq\left[\theta_{M}, \omega_{M}, \theta_{L}, \omega_{L}\right]^{T}
$$

${ }^{1}$ The physical cause of this mismatch is not clear to the authors at this moment. This will be the topic of future research. 


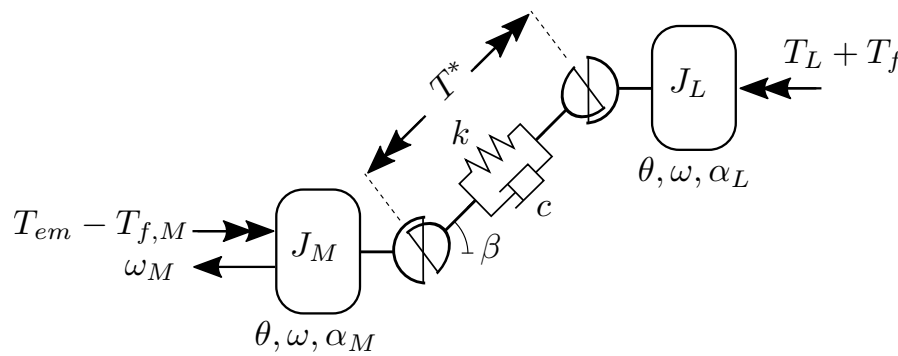

Fig. 1. Schematic view on the mechanical driveline model.

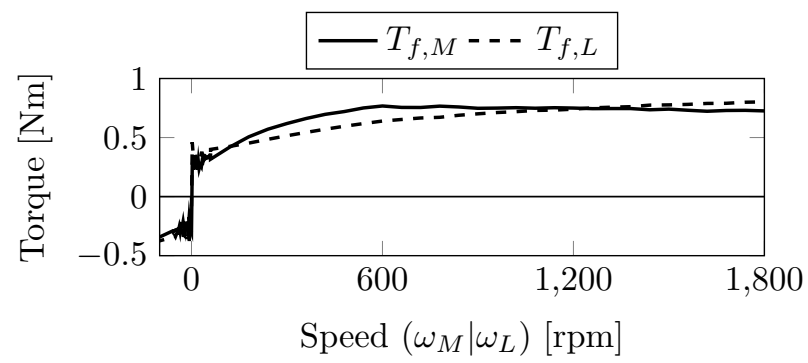

Fig. 2. Experimentally identified friction torques.

The rotational speeds and angles are related to the mechanical state variables through the cardan joint kinematic relations [27]

$$
\begin{aligned}
\theta_{j}^{*} & =\tan ^{-1}\left(\cos \beta \tan \theta_{j}\right), \\
\omega_{j} & =\omega_{j}^{*}\left[\cos \beta\left(\cos \theta_{j}\right)^{2}+\frac{1}{\cos \beta}\left(\sin \theta_{j}\right)^{2}\right] .
\end{aligned}
$$

The torques exerted by the cardan transmission onto the motor and load are denoted $T_{M}^{c}$ and $T_{L}^{c}$. These follow from the shaft torque (6) by assuming lossless torque transfer:

$$
T_{j}^{c}=T^{*} \frac{\omega_{j}^{*}}{\omega_{j}}, \quad j=M, L .
$$

The kinematics for this system are nonlinear at nonzero deflection angles $\beta=\tan ^{-1}(\tau)$. The dimensionless parameter $\tau$ denotes the ratio of lateral over axial distance between the center points of both cardan joints. Nonlinearity in the torsional dynamic behavior arises whenever $\tau \neq 0$.

In addition to the electromagnetic torque $T_{e m}$ and the load torque input $T_{L}$, the proposed model contains friction torques $T_{f, M}$ and $T_{f, L}$. These are obtained as function of speed through a table-based interpolation based on an offline identification for the system under investigation. Figure 2 shows the steady-state operating points that were identified. The model is evaluated in state-space according to (11) by substituting the joint torque from (10) in the dynamic force balance equations (12):

$$
\begin{aligned}
\frac{d \mathbf{x}_{m}}{d t} & =\left[\omega_{M}, \alpha_{M}, \omega_{L}, \alpha_{L}\right]^{T} \\
{\left[\begin{array}{c}
J_{M} \alpha_{M} \\
J_{L} \alpha_{L}
\end{array}\right] } & =\left[\begin{array}{c}
T_{e m}-T_{f, M}-T_{M}^{c} \\
T_{L}^{c}-T_{f, L}-T_{L}
\end{array}\right]
\end{aligned}
$$

With the above equations, the mechanical drivetrain submodel is fully defined and can be coupled to the $\mathrm{AC}$ induction machine model.

\section{Mechatronic powertrain model}

The mechatronic powertrain model results from coupling the lumped electrical model with the mechanical model. The resulting state vector $\mathbf{x}$ is composed as:

$$
\mathbf{x}=\left[\begin{array}{c}
\mathbf{x}_{e l} \\
\mathbf{x}_{m}
\end{array}\right]
$$

The known inputs $\mathbf{u}$ to this model are the stator voltages $\left[u_{\alpha}, u_{\beta}\right]$. In this work, the load torque $T_{L}$ is treated as an unknown input $u$. In order to enable the estimation of this input in a global state-estimation framework, it is added as an augmented state. In addition to the state variables, predictions of the stator currents $\left[i_{\alpha}, i_{\beta}\right]$ and accelerations at motor and load side $\left[\alpha_{M}, \alpha_{L}\right]$ are also available as model outputs.

\section{Sensor Selection Analysis}

For accurate estimation, an informative set of measurements $\mathbf{y}$ is required in addition to an accurate model. The continuoustime system model and measurements equations are written as:

$$
\begin{aligned}
\dot{\mathbf{x}} & =\mathbf{f}(\mathbf{x}, u)+\mathbf{g}(\mathbf{u}) \\
\mathbf{y} & =\mathbf{h}(\mathbf{x}, u) .
\end{aligned}
$$

with the (continuous) state space update function composed of a drift vector field $\mathbf{f}$ and a control vector field $g$ and with measurement function $\mathbf{h}$. Preferably the number and cost of sensors is kept as low as possible. The minimum sensor selection is addressed by observability theory. The observability analysis allows to determine whether it is theoretically possible to determine the states of the system from a set of measurements. A second aspect which is particularly important in the case of highly dynamic estimation, is the bandwidth of the filter. Both aspects are discussed for the coupled state/input estimation in a mechatronic powertrain.

\section{A. Model augmentation for coupled state/input estimation}

One method for concurrent estimation of an unknown load with the regular model states is by adding the unknown input state $u \in \mathbb{R}^{1}$ to an augmented state vector $\mathbf{x}_{a} \in \mathbb{R}^{n+1}$. In this case, a model $f_{u}$ has to be provided for the input state and added to the system model:

$$
\begin{aligned}
& \mathbf{x}_{a} \triangleq\left[\begin{array}{c}
\mathbf{x} \\
u
\end{array}\right] \\
& \mathbf{f}_{a} \triangleq\left[\begin{array}{r}
\mathbf{f}\left(\mathbf{x}_{a}\right) \\
f_{u}\left(\mathbf{x}_{a}\right)
\end{array}\right] .
\end{aligned}
$$

In contrast to the state update model in (14), the informative value of $f_{u}$ is usually limited. A correspondingly high model uncertainty will therefore be attributed to the latter, such that the estimator will depend heavily on the measurements to correct the prediction of $u$. The precise definition of $f_{u}$ thus has limited influence on the estimation result and a first order random walk model is often a suitable choice:

$$
\dot{u}=\dot{x}_{n+1}=0+w_{\dot{u}}(t)
$$

with $w_{\dot{u}}(t)$ a zero-mean white noise process with power spectral density $Q_{\dot{u} \dot{u}}$. On average this implies that the load should remain constant, but by accounting for a high covariance in the corresponding discrete-time noise model (see section IV) it allows to track fast and high variations in an unknown process. 


\section{B. Practical measurement sets}

A number of practically achievable measurement sets are selected for the mechatronic powertrain investigated in this work. They are summarized in Table I. These measurement

TABLE I

Candidate Measurement Sets

\begin{tabular}{c||cccc}
\hline \hline $\mathbf{y}_{(. .)} \in \mathbb{R}^{5}$ & $\mathbf{y}_{\omega \omega}$ & $\mathbf{y}_{\alpha \omega}$ & $\mathbf{y}_{\omega \alpha}$ & $\mathbf{y}_{\alpha \alpha}$ \\
\hline \hline$\left[y_{1}, y_{2}, y_{3}\right]^{T}$ & & {$\left[i_{s \alpha}, i_{s \beta}, \theta_{M}\right]^{T}$} & \\
\hline$y_{4}$ & $\omega_{M}$ & $\alpha_{M}$ & $\omega_{M}$ & $\alpha_{M}$ \\
$y_{5}$ & $\omega_{L}$ & $\omega_{L}$ & $\alpha_{L}$ & $\alpha_{L}$ \\
\hline \hline
\end{tabular}

sets will be evaluated, both theoretically in this section and experimentally in section $\mathrm{V}$, for their observability and estimator bandwidth in order to select the most suitable set. We also perform the theoretical observability analysis for the case where no measurement of the rotor angle $\theta_{M}$ is available. This is denoted as $\mathbf{y}_{(. .)} \backslash \theta_{M}$ and could be particularly interesting for applications with 'sensorless' IM control.

\section{Observability analysis}

Matrix rank criteria are the most common methods for assessing the observability properties of a certain modelsensor combination. Many authors have already studied these criteria for both linear and nonlinear models , as well as their respective equivalence ( [17]-[19], [28]). In the following, we focus on the assessment of the observability through the rank of the Lie-derivative matrix of the measurement equations. The subscript $\cdot{ }_{a}$ is omitted from here onwards, i.e. $\mathbf{x}_{a}$ and $\mathbf{f}_{a}$ are denoted as $\mathbf{x}$ and $\mathbf{f}$, respectively.

The observation space $\mathcal{O}_{k}$ reduces to the span of the Lie derivatives $\mathcal{L}_{\mathbf{f}}^{0} \mathbf{h}, \ldots, \mathcal{L}_{\mathbf{f}}^{k-1} \mathbf{h}$ with respect to the drift vector field $\mathbf{f}$ :

$$
\mathcal{O}_{k}=\operatorname{span}\left\{\mathbf{h}, \mathcal{L}_{\mathbf{f}}^{1} \mathbf{h}, \ldots, \mathcal{L}_{\mathbf{f}}^{k-1} \mathbf{h}\right\} .
$$

The observability matrix $\mathrm{d} \mathcal{O} \triangleq \partial \mathcal{O} / \partial \mathbf{x}$ with rank equal to the dimension of the tangent space of $\mathcal{O}_{k}$ is then obtained as

$$
d \mathcal{O}_{k}=\frac{\partial}{\partial \mathbf{x}}\left[\begin{array}{c}
\mathcal{L}_{\mathbf{f}}^{0} \mathbf{h} \\
\mathcal{L}_{\mathbf{f}}^{1} \mathbf{h} \\
\vdots \\
\mathcal{L}_{\mathbf{f}}^{k-1} \mathbf{h}
\end{array}\right]
$$

computing $j$-th order Lie derivatives recursively:

$$
\mathcal{L}_{\mathbf{f}}^{0} \mathbf{h}=\mathbf{h}(\mathbf{x}), \quad \mathcal{L}_{\mathbf{f}}^{j} \mathbf{h}=\frac{\partial}{\partial \mathbf{x}}\left(\mathcal{L}_{\mathbf{f}}^{j-1} \mathbf{h}\right) \mathbf{f}(\mathbf{x}) \forall j>0 .
$$

A sufficient condition for the augmented system (14)-(16) to be weakly locally observable is that its observability matrix is of full rank $n+1$.

In order for the Kalman filter to guarantee convergence, the system is required to be weakly locally observable for arbitrary control input $\mathbf{u}^{2}$. This is the case if this observability matrix is of full rank.

\footnotetext{
${ }^{2}$ This is not a sufficient condition as also the filter tuning has an impact on the convergence.
}

TABLE II

OBSERVABILITy MATRIX RANK EVALUATION

\begin{tabular}{c||cccc|cccc}
\hline \hline \multicolumn{1}{l|}{} & \multicolumn{4}{c|}{$\mathbf{y}_{2} \mathbf{y}_{(. .)} \in \mathbb{R}^{5}$} & \multicolumn{4}{c}{$\mathbf{y}=\mathbf{y}_{(. .)} \backslash \theta_{M} \in \mathbb{R}^{4}$} \\
rank & $\mathbf{y}_{\omega \omega}$ & $\mathbf{y}_{\alpha \omega}$ & $\mathbf{y}_{\omega \alpha}$ & $\mathbf{y}_{\alpha \alpha}$ & $\mathbf{y}_{\omega \omega}$ & $\mathbf{y}_{\alpha \omega}$ & $\mathbf{y}_{\omega \alpha}$ & $\mathbf{y}_{\alpha \alpha}$ \\
\hline \hline $\mathrm{d} \mathcal{O}_{0}$ & 5 & 5 & 5 & 5 & 4 & 4 & 4 & 4 \\
$\mathrm{~d} \mathcal{O}_{1}$ & $\mathbf{9}$ & $\mathbf{9}$ & $\mathbf{9}$ & $\mathbf{9}$ & 8 & 8 & 8 & 8 \\
$\mathrm{~d} \mathcal{O}_{2}$ & 9 & 9 & 9 & 9 & $\mathbf{9}$ & $\mathbf{9}$ & $\mathbf{9}$ & $\mathbf{9}$ \\
\hline \hline
\end{tabular}

The observability matrix is evaluated according to (18) for the system model with the first order random walk input model for each of the four different candidate measurement sets $\mathbf{y}_{(. .)}$listed in Table I, with and without $\theta$-measurement. The augmented system has a total of $n+1=9$ states to estimate. Table II lists the rank of the observability matrices $\mathrm{d} \mathcal{O}_{k}$. The column vector $\mathcal{O}_{k}$ represents the considered subspace of the observation space $\mathcal{O}$ of $\mathbf{Y}$, taking into account the measured values and their time-derivatives up to order $k$. Bold values indicate weak local observability, i.e. a full rank for $\mathrm{d} \mathcal{O}_{k}$. This analysis indicates that all possible measurement sets lead to an observable system and theoretically enable a stable estimator.

It is also noteworthy that no explicit angle measurement is required. This is in contrast to when a purely mechanical model is used. The motor torque would then become a second unknown input and system observability would require two shaft angle sensors [29]. These would have to be very accurate, therefore it is advisable to include the electrical motor model and employ the electrical measurements.

In the following section we present a bandwidth analysis in order to evaluate the capability of different sensor sets to track dynamic behavior.

\section{Bandwidth analysis}

A second aspect which is important in the sensor selection for an estimator, is the desired bandwidth. This aspect is less frequently discussed for Kalman filter based techniques, even though some authors have investigated it for the linear continuous-time Kalman filter [30]. Evaluating the theoretical frequency response for the linearized powertrain and the steady-state Kalman gain yields valuable insight in the impact of the sensor selection on the bandwidth of the final estimator.

Fig. 3 summarizes the frequency response magnitudes for the unknown input estimation with the four measurement sets from Table I with the angle measurement ${ }^{3}$. This figure clearly shows that, for exactly the same model covariance on the random walk input model, the estimator bandwidth can be increased drastically by adding an accelerometer on the load side. The lower bandwidth obtained when the accelerometer is put on the motor side can be attributed to the filtering behavior of the mechanical system between the load and the motor. These theoretical characteristics still assume perfect sensor behavior, in the sense that they do not account for the lower practical bandwidth of the rotational velocity sensors with

\footnotetext{
${ }^{3}$ This figure is set up using the stochastic parameters discussed in the Appendix $\mathrm{A}$ and for the aligned driveline configuration, i.e. $\tau=0$.
} 


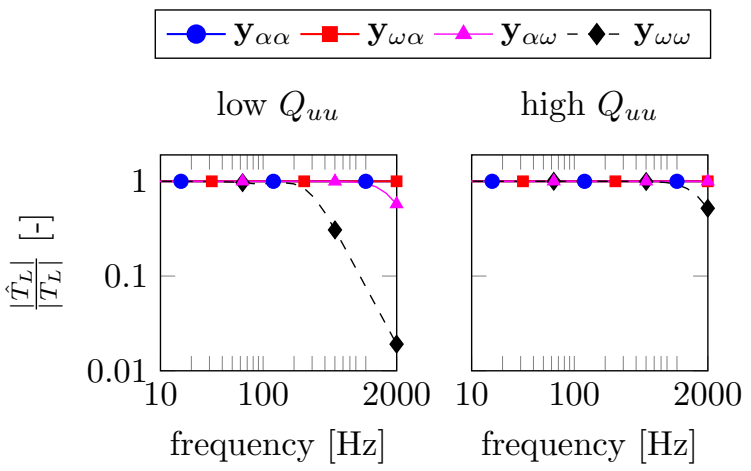

Fig. 3. Linearized closed-loop estimator behavior for low and high input model uncertainty $Q_{u u^{3}}{ }^{3}$ : theoretical attenuation $|\hat{u}| /|u|=\left|\hat{T}_{L}\right| /\left|T_{L}\right|$ for sensor sets $\mathbf{y}_{\alpha \alpha}, \mathbf{y}_{\omega \alpha}, \mathbf{y}_{\alpha \omega}$ and $\mathbf{y}_{\omega \omega}$.

respect to Ferraris sensors and the low-pass filtering performed on the Ferraris sensors. They also assume modeling errors to not depend on the frequency content of the input. Experimental results in the case of broadband input are presented in section $\mathrm{V}$ and support the results from this theoretical analysis.

\section{Coupled State/Input Estimation}

The proposed virtual torque sensor follows the approach of coupled state/input estimation. The state vector of (13) corresponds to the model of section II. It is augmented to $\mathbf{x}_{a}$ as in (15), where $u$ is the unknown load torque $T_{L}$. In the following of this paper, the subscript $\cdot_{a}$ is omitted and $\mathbf{x}$ denotes the augmented state vector. Online estimation of the augmented state vector is done by means of a recursive Kalman filter (KF).

First a prediction step requires coupling the mechatronic powertrain and input model of (14) and (16) into the discrete time formulation of (19). In the here used Amesim modeling environment, the discretization is handled by Amesim's builtin variable step-size integrator. The evaluation of $\mathbf{f}_{d}$ thus consists of (i) performing a black-box integration procedure from time $t_{k-1}$ to $t_{k}$ on the model of section II and (ii) of setting $u_{k}=u_{k-1}$. The measurement function $\mathbf{h}$ depends on the selected measurement set from Table I and is also readily evaluated using the Amesim model. The model noise $\mathbf{w}_{k}$ and measurement noise $\mathbf{v}_{k}$ are assumed to have zero-mean Gaussian probability density functions and are characterized by their respective covariance matrices $\mathbf{Q}$ and $\mathbf{R}$.

$$
\begin{array}{ll}
\mathbf{x}_{k}=\mathbf{f}_{d}\left(\mathbf{x}_{k-1}, \mathbf{u}_{k-1}\right) & +\mathbf{w}_{k} \\
\mathbf{y}_{k}=\mathbf{h}\left(\mathbf{x}_{k}, \mathbf{u}_{k}\right) & +\mathbf{v}_{k} .
\end{array}
$$

In any KF approach [8]-[10], the model prediction is followed by an update step (20). The differences between the measurements and their predictions $\left(\mathbf{y}_{k}-\hat{\mathbf{y}}_{k}^{-}\right)$, called innovation, are considered to improve the estimate of $\mathbf{x}_{k}$ and reduce the state-covariance matrix $\mathbf{P}_{x x}$ :

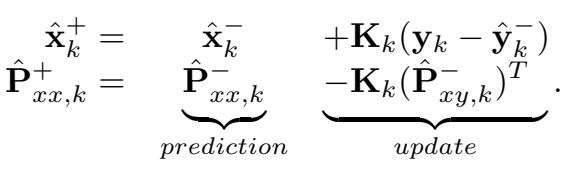

In these equations, the superscripts $\cdot^{-}$and ${ }^{+}$indicate socalled a-priori model predictions and a-posteriori estimates, respectively. The Kalman gain matrix $\mathbf{K}_{k}$ weights the crosscovariance $\mathbf{P}_{x y}$ between states and innovation against the innovation auto-covariance $\mathbf{P}_{y y}$ :

$$
\mathbf{K}_{k}=\hat{\mathbf{P}}_{x y, k}^{-}\left(\hat{\mathbf{P}}_{y y, k}^{-}\right)^{-1} .
$$

The Extended Kalman filter (EKF) and the Unscented Kalman filter (UKF) employ different approaches in evaluating (20)(21). A detailed discussion on how the choice between the EKF and UKF affects estimator performance can be found in literature [15], [31]. This work regards both KF variants and their differences from a practical perspective, as section $\mathrm{V}$ will show that the virtual torque sensor performance primarily depends on the considered measurement set.

\section{A. Extended Kalman filter}

The EKF [10] yields a-priori states and measurements at time $t_{k}$ by evaluating the discrete model of (19). It disregards any stochastic aspect due to error terms $\mathbf{w}_{k}$ and $\mathbf{v}_{k}$.

$$
\begin{aligned}
& \hat{\mathbf{x}}_{k}^{-}=\mathbf{f}_{d}\left(\hat{\mathbf{x}}_{k-1}^{+}, \mathbf{u}_{k-1}\right) \\
& \hat{\mathbf{y}}_{k}^{-}=\mathbf{h}\left(\hat{\mathbf{x}}_{k}^{-}, \mathbf{u}_{k}\right)
\end{aligned}
$$

The state and innovation auto-covariance matrices are then approximated by linearizing the system, respectively around $\left(\hat{\mathbf{x}}_{k-1}^{+}, \mathbf{u}_{k-1}\right)$ and $\left(\hat{\mathbf{x}}_{k}^{-}, \mathbf{u}_{k}\right)$.

$$
\begin{array}{lll}
\hat{\mathbf{P}}_{x x, k}^{-}=\mathbf{Q}+\mathbf{F} \hat{\mathbf{P}}_{x x, k-1}^{+} \mathbf{F}^{T} & \mathbf{F}=\frac{\partial \mathbf{f}_{d}}{\partial \mathbf{x}} \\
\hat{\mathbf{P}}_{y y, k}^{-}=\mathbf{R}+\mathbf{H} \hat{\mathbf{P}}_{x x, k}^{-} \mathbf{H}^{T} & \mathbf{H}=\frac{\partial \mathbf{h}}{\partial \mathbf{x}}
\end{array}
$$

Evaluation of (20)-(21) is done by further employing the linearized measurement function $\mathbf{H}$.

$$
\hat{\mathbf{P}}_{x y, k}^{-}=\hat{\mathbf{P}}_{x x, k}^{-} \mathbf{H}^{T}
$$

The jacobian matrices $\mathbf{F}$ and $\mathbf{H}$ in (23)-(24) can be evaluated efficiently using the Amesim model of section II, as the Amesim modeling tool features jacobian evaluations by means of internal numerical differentiation. This allows the implemented EKF algorithm to run approximately four times faster than the UKF algorithm discussed below, which is in contrast to other implementations described in literature [32]. In case of strongly nonlinear dynamics or discontinuities, the use of only first-order approximations may also adversely affect estimator performance [15]. These are the main reasons why the UKF may be preferred over the EKF.

\section{B. Unscented Kalman filter}

The UKF [14], [15] is named after its use of the Unscented Transformation (UT) [13]. The UT approximates a nonlinearly transformed distribution's mean and covariance values by evaluating the nonlinear function in a set of sigma-points $\chi$ around the expected value and weighting the results according to the assumed distribution. Through successive evaluation of (25)-(28) , the UKF applies this principle to obtain the a-priori 
estimate $\hat{\mathbf{x}}_{k}^{-}$and its covariance $\hat{\mathbf{P}}_{x x, k}^{-}$based on the a-posteriori estimate and its covariance at the previous time $t_{k-1}$.

$$
\begin{aligned}
& \chi_{k-1}=\left[\begin{array}{lll}
\hat{\mathbf{x}} & \hat{\mathbf{x}}+\sqrt{n \hat{\mathbf{P}}_{x x}} & \hat{\mathbf{x}}-\sqrt{n \hat{\mathbf{P}}}
\end{array}\right]_{k-1}^{+} \\
& \chi_{i}^{*}=\mathbf{f}_{d}\left(\chi_{i, k-1}, \mathbf{u}_{k-1}\right) \\
& \hat{\mathbf{x}}_{k}^{-}=\sum W_{i} \chi_{i}^{*} \\
& \hat{\mathbf{P}}_{x x, k}^{-}=\mathbf{Q}+\sum W_{i}\left(\chi_{i}^{*}-\hat{\mathbf{x}}_{k}^{-}\right)\left(\chi_{i}^{*}-\hat{\mathbf{x}}_{k}^{-}\right)^{T}
\end{aligned}
$$

The implementation of (25) is valid in the case of additive model and measurement noise ${ }^{4}$. All weights are set to $W_{i}=$ $1 / 2 n$ with $n$ the number of states, except for $W_{0}=0$ so that only $2 n$ sigma points are actually used.

Applying the same approach to the newly obtained a-priori estimate and replacing $\mathbf{f}_{d}$ with $\mathbf{h}$ in (26) yields $\hat{\mathbf{y}}_{k}^{-}$. The covariance matrix $\hat{\mathbf{P}}_{y y, k}^{-}$is found by replacing $\mathbf{Q}$ with $\mathbf{R}$ and $\hat{\mathbf{x}}_{k}^{-}$with $\hat{\mathbf{y}}_{k}^{-}$in (28). The cross-covariance $\hat{\mathbf{P}}_{x y, k}^{-}$is then computed as in (29).

$$
\hat{\mathbf{P}}_{x y, k}^{-}=\sum W_{i}\left(\chi_{i, k}-\hat{\mathbf{x}}_{k}^{-}\right)\left(\chi_{i}^{*}-\hat{\mathbf{y}}_{k}^{-}\right)^{T}
$$

Similar as in the EKF scheme, the Kalman gain and update can then be evaluated according to the general expressions (20)(21). Unlike in the EKF scheme, jacobian evaluations are not required. The UKF's prediction step does require $2 n$ model integration steps (26), whereas the EKF prediction is based on a single integration step.

\section{EXPERIMENTAL VALIDATION}

The proposed virtual load torque sensor makes use of the mechatronic model from section II and the measurement set $\mathbf{y}_{\alpha \alpha}$ as defined in section III. A comparison is made between this and the other measurement sets and between the EKF and UKF implementations discussed in section IV. The used model parameter values and filter settings are given in Appendix A. The following three sub-sections describe the experimental test setup used for validation and discuss estimator performance during transient operation and for broadband excitation.

\section{A. Experimental test setup}

The mechatronic powertrain test setup is shown in Fig. 4. This test setup is purpose-built and instrumented for the validation of virtual sensors based on physical powertrain models [22]. It consists of two $5.5 \mathrm{~kW}$ asynchronous IM that are connected mechanically by a double cardan transmission in series with an HBM T40B torque sensor. The IM at the left side, denoted $M$, can be displaced laterally. The cardan assembly is then Z-configured, i.e. $\tau>0$, and introduces nonlinear torsional dynamics. Fig. 4 also indicates the modeled part of the powertrain, the reference torque measurement $T_{L}$ and the measurements for estimation.

Estimator performance under transient operation will be discussed first. To this end, the motor speed $\omega_{M}$ is controlled towards a linear run-up while the electromagnetic reference torque at the load side $T_{e m, L}^{r e f}$ is maintained constant at $5 \mathrm{Nm}$. This operating trajectory has been imposed twice, with the

${ }^{4}$ Otherwise the sigma point vectors should be augmented [15].

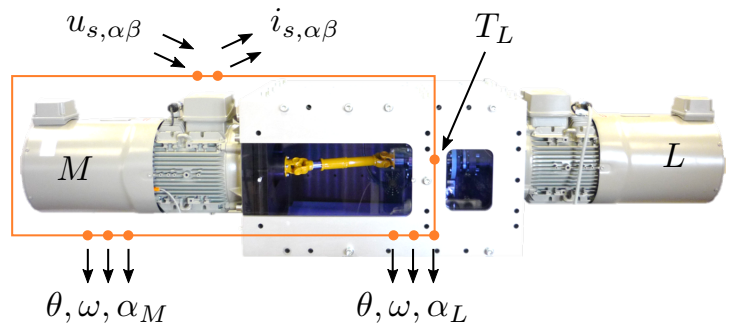

Fig. 4. Experimental test setup. The indicated measurements correspond to the model inputs and outputs, cfr. section II.

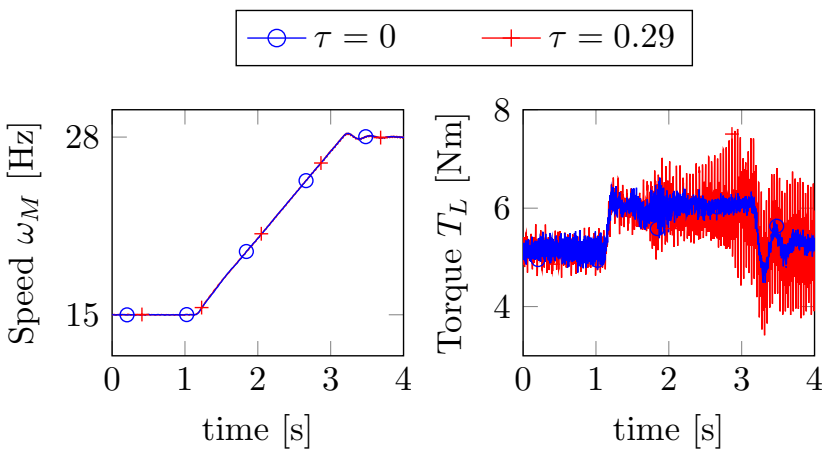

Fig. 5. Measured motor speed $\omega_{M}$ and load torque $T_{L}$ for both run-ups at constant electromagnetic load torque reference $T_{e m, L}^{r e f}=5 \mathrm{Nm}$.

cardan driveline aligned $(\tau=0)$ in one case and Z-configured $(\tau=0.29)$ in the other.

Fig. 5 shows the measured speed $\omega_{M}$ (derived from the same 1024-pulse encoder that yields the angle measurement $\theta_{M}$ ) and load torque $T_{L}$ during each of these two cases. Torsional dynamic behavior is clearly excited more prominently in the misaligned case. Estimation results will further be discussed for this run-up with $\tau=0.29$.

The estimator bandwidth has been assessed by means of a second set of two validation cases, again respectively in the aligned configuration and with $\tau=0.29$. The motor speed reference is here maintained at $\omega_{M}^{r e f}=15 \mathrm{~Hz}$, while $T_{e m, L}^{r e f}$ is now such that the load torque input consists primarily of multi-sine excitation:

$$
T_{L} \approx 5 \mathrm{Nm}+\sum_{i=1}^{8} A_{i} \sin \left(2 \pi f_{i}+\phi_{i}\right) .
$$

The excitation frequencies $f_{i}$ are listed in Table III, together with their corresponding amplitudes $A_{i}$ and phase angles $\phi_{i}$. They excite the system below, at, and above its torsional driveline resonance at $78 \mathrm{~Hz}^{5}$.

\section{B. Estimator results under transient operation}

First, the combination of the proposed measurement set $\mathbf{y}_{\alpha \alpha}$ with the mechatronic powertrain model of section II is considered under the transient operation with misalignment.

\footnotetext{
${ }^{5}$ These frequencies correspond to $f_{i}=6 \mathrm{~Hz} \times(1+4 k), k \geq 0$. The frequency of $174 \mathrm{~Hz}$, corresponding to $k=7$, is nearby a system zero. It is thus difficult to excite in a well-defined manner and therefore this frequency is excluded from the multi-sine.
} 

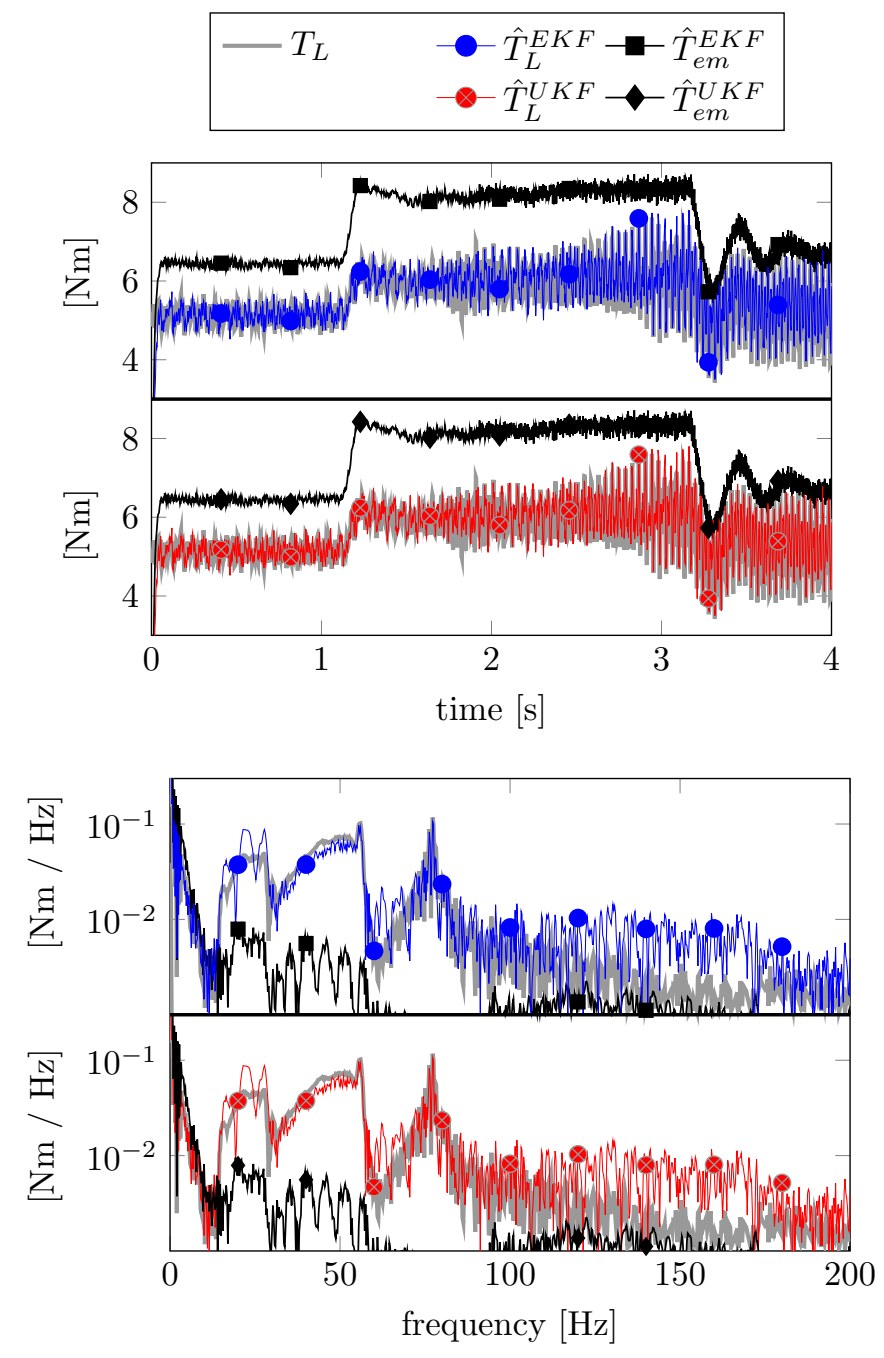

Fig. 6. Measured load torque $T_{L}$, EKF and UKF estimates $\hat{T}_{L}$ and $\hat{T}_{e m}=\hat{T}_{e m, M}$, during the run-up of the misaligned driveline.

Fig. 6 shows the resulting EKF and UKF torque estimates in the time and frequency domain. The spectral densities (SD) shown here and in the following result from a fast fourier transformation on the hanning-windowed time data from $\mathrm{t}=0.1 \mathrm{~s}$ to $\mathrm{t}=4.1 \mathrm{~s}$ and applying the corresponding amplitude correction factor.

The misaligned configuration yields prominent excitation at the second shaft order, i.e. from 30 to $56 \mathrm{~Hz}$. Even multiples of this shaft order pass by the first torsional eigenfrequency at $78 \mathrm{~Hz}$, such that this resonance is clearly visible in the SD plot as well. The measured and estimated load torque clearly correspond well in the excited frequency range.

Apart from their transient at start-up, EKF and UKF results are nearly identical, despite the nonlinear nature of the considered estimation problem. This may be explained by the small timestep of $0.25 \mathrm{~ms}$, which is required for accurate estimation and justifies the EKF's local linearization approach ${ }^{6}$.

\footnotetext{
${ }^{6}$ The filter timestep has been increased to $4 \mathrm{~ms}$ with no divergence problems. Estimation accuracy is then reduced drastically, though, by lack of sufficient measurement information. This effect is much more prominent than the emerging difference between the EKF and the UKF.
}

In addition to the estimated load torque $\hat{T}_{L}$, Fig. 6 also shows the estimated electromagnetic torque $\hat{T}_{e m}$ exerted by the motor $M$. The latter differs considerably from the load torque $\hat{T}_{L}$ at the other side of the modeled driveline. This highlights the inadequacy of using only electrical motor measurements to estimate dynamic load torques in mechatronic applications.

The difference is partly caused by the presence of friction in the driveline's bearings. Due to the driveline's inertia, the mismatch grows during speed transients. More importantly, the load torque components at frequencies above $10 \mathrm{~Hz}$ are transmitted to the electrical domain only partly. This is due to the inherent low-pass filtering effect of any torsionally flexible driveline that connects the electrical motor to the load. Due to this, knowledge of the electromagnetic torque $T_{e m, M}$ suffices to identify the load torque $T_{L}$ accurately only in the frequency band where the driveline dynamics are not excited. This leads to the rule of thumb that an electrical IM model suffices if the estimation bandwidth of interest does not exceed $f_{\text {res }} / 10$, with $f_{\text {res }}$ the lowest torsional eigenfrequency of the driveline. For accurate load torque estimation over a wider frequency band, the dynamic mechanical behavior should be included in the model. As noted in Section III, the electrical model is still required in this case.

\section{Estimator bandwidth}

Fig. 7 shows the main practical significance of section III, by comparing the estimation results for different measurement sets of table I. Two sets of results are shown. These both correspond to the validation case of multisine excitation and $\tau=0.29$. Their difference is caused by attributing a different amount of uncertainty to the input model $u_{k+1}=u_{k}$. As the latter is quantified by the diagonal element $Q_{u u}$ of the covariance matrix $\mathbf{Q}$, the results are denoted as low $Q_{u u}$ and high $Q_{u u}$, respectively. The time-domain results are zoomed and switch from low to high $Q_{u u}$ at $t=2.55 \mathrm{~s}$. The spectral density plots zoom in on $f_{i}=150 \mathrm{~Hz} \approx 2 f_{\text {res }}$.

The measurement set $\mathbf{y}_{\alpha \alpha}$ yields a torque estimate that follows transients well and is not at all sensitive to the setting of the input model covariance $Q_{u u}$. Using a motor speed measurement instead of acceleration yields $\mathbf{y}_{\omega \alpha}$. When assuming the lower input model uncertainty, this measurement set leads to attenuated high-frequency components in the estimate. The effect is even more severe when using $\mathbf{y}_{\omega \omega}$, i.e. speed is measured instead of acceleration at both sides of the driveline. The set $\mathbf{y}_{\alpha \omega}$ yields significantly better results than $\mathbf{y}_{\omega \alpha}$. This is true for this particular driveline, in which the rotational inertia at load side is far smaller than that of the motor. Because of this, the speed at load side reacts much faster to torque transients. However, the obtainable virtual sensor bandwidth is still much broader when using $\mathbf{y}_{\alpha \alpha}$.

When assuming the higher input model uncertainty, the lack of high-frequency information in the measurement sets $\mathbf{y}_{\alpha \omega}$ and $\mathbf{y}_{\omega \omega}$ seems to be countered by allowing a more aggressive correction on the model prediction of the load torque. However, the time- and frequency-domain results show that this is at the cost of a much bigger overall error. 

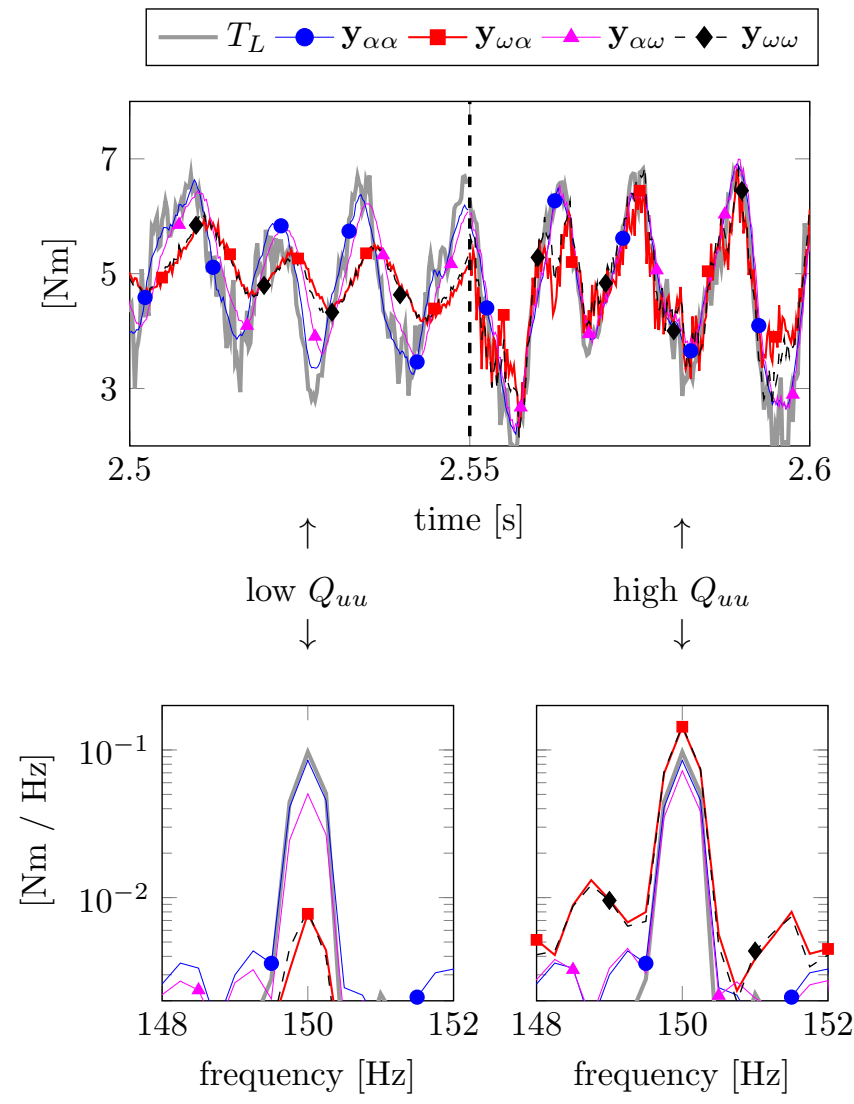

Fig. 7. Measured load torque $T_{L}$ and EKF estimate $\hat{T}_{L}$ for measurement sets $\mathbf{y}_{\alpha \alpha}, \mathbf{y}_{\omega \alpha}, \mathbf{y}_{\alpha \omega}$ and $\mathbf{y}_{\omega \omega}$, assuming (left) low and (right) high uncertainty on the input model. Zoom of time-domain results and spectral densities for the misaligned driveline under multisine excitation.

Finally, the accuracy of the proposed virtual torque sensor has been assessed in detail for both validation cases with multisine excitation. Table III summarizes the estimation errors in terms of amplitude attenuation and phase difference with respect to the reference measurement $T_{L}$. The attenuation is significantly lower than $1 \mathrm{~dB}$ for most frequencies, and exceeds $3 \mathrm{~dB}$ only at $198 \mathrm{~Hz}$. The phase difference is limited to $10^{\circ}$ in both configurations and it is much smaller at almost all excited frequencies. In addition to this table, the bias has been verified to be less than $0.04 \mathrm{Nm}$ in both configurations. The RMSE amounts to $0.33 \mathrm{Nm}$ for the case where $\tau=0$ and $0.36 \mathrm{Nm}$ for $\tau=0.29$. When applying a low-pass filter with a cut-off frequency of $250 \mathrm{kHz}$ on the measured torque $T_{L}$, these RMSE reduce to $0.17 \mathrm{Nm}$ and $0.21 \mathrm{Nm}$, respectively.

\section{CONCLUSION}

This paper has proposed a virtual torque sensor that yields online and broadband load torque estimation on a mechatronic powertrain. It has contributed to the field of non-invasive torque estimation by showing the benefit of modeling both the electrical and the mechanical dynamics and by relating available instrumentation to input estimation results through nonlinear observability theory and an estimator bandwidth analysis.
TABLE III

EKF RESULTS BASED ON $\mathbf{y}_{\alpha \alpha}$

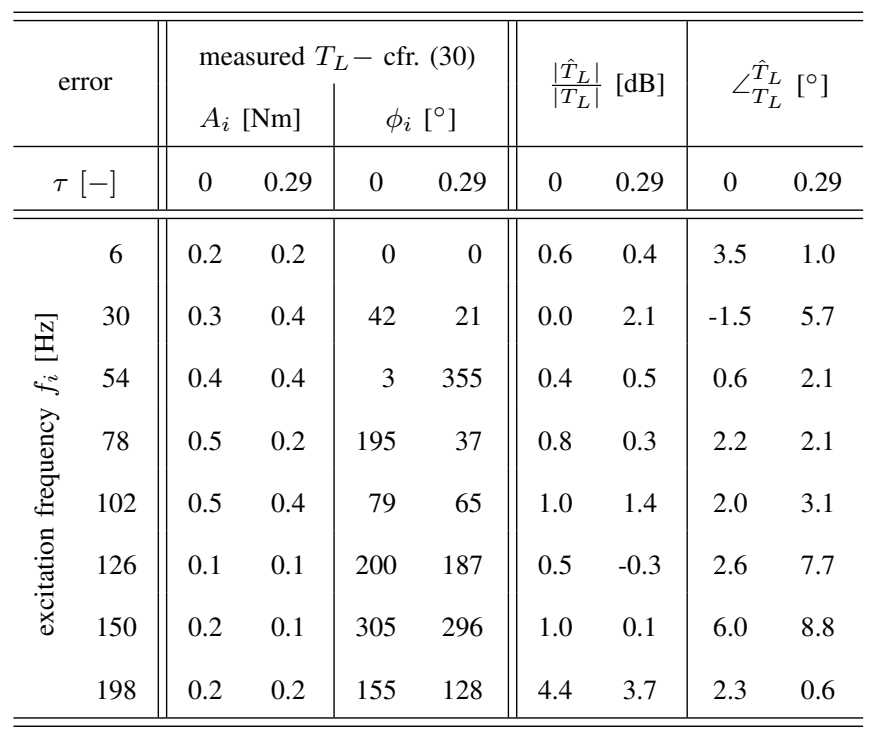

The proposed approach is based on a nonlinear torsional powertrain model and measurements of the motor terminal voltages and currents, an encoder angle and two rotational accelerations. It has been validated experimentally, on a powertrain that consists of an IM and a double cardan transmission. This validation includes a comparison between the use of an augmented EKF and an augmented UKF, as well as between four possible measurement sets.

Fusion of the model with the measurements may be done with either the EKF or the UKF, as both yield almost indistinguishable results. However, it has been shown theoretically and experimentally that the inclusion of both acceleration measurements is critical for the performance of the online estimator. For low-bandwidth applications, one or both acceleration measurements may be replaced with speed measurements.

The accuracy of the proposed virtual torque sensor has been verified under transient speed and torque conditions for an aligned and Z-configured double cardan transmission. The Z-configuration introduces significantly nonlinear dynamics. The virtual sensing accuracy has been quantified by means of multi-sine excitation. Accurate results are obtained in the frequency region from $0 \mathrm{~Hz}$ to $200 \mathrm{~Hz}$. The proposed approach is thus valid over a bandwidth that covers regions below, around, and above the first torsional eigenfrequency at $78 \mathrm{~Hz}$.

\section{APPENDIX A ESTIMATOR SETTINGS}

All presented estimation results have been generated with a filter time step of $0.25 \mathrm{~ms}$. This equals the sampling period of the measurements fed into the estimator. The highest frequency in Fig. 3 is the corresponding Nyquist frequency of $2 \mathrm{kHz}$.

The electrical model parameter values are $r_{s}=0.82 \Omega$, $l_{\sigma s}=0.014 \mathrm{H}, L_{m}=0.17 \mathrm{H}$ and $\tau_{r}=0.74 \mathrm{~s}$. The mechanical model parameter values are $J_{M}=0.016 \mathrm{~kg} \cdot \mathrm{m}^{2}$, $J_{L}=0.0025 \mathrm{~kg} \cdot \mathrm{m}^{2}, k=34 \mathrm{Nm} /{ }^{\circ}$ and $c=0.12 \mathrm{Nm} / \mathrm{rpm}$. 
The state update covariance matrix is determined as indicated in Appendix B. In SI units, it amounts to

$$
\begin{aligned}
\mathbf{Q}=\operatorname{diag}(1.7 \mathrm{e}-4,1.7 \mathrm{e}-4,4 \mathrm{e}-8,4 \mathrm{e}-8, \\
\left.8 \mathrm{e}-8,2.4 \mathrm{e}-3,8 \mathrm{e}-8,4.4 \mathrm{e}-3, Q_{\text {uи }}\right),
\end{aligned}
$$

where $Q_{u u}=38 \mathrm{Nm}^{2}$ (denoted as high $Q_{u u}$ ) or $Q_{u u}=0.038 \mathrm{Nm}^{2}$ (denoted as low $Q_{u u}$ ).

The measurement covariance matrix is based on sensor datasheets. In SI units and for $\mathbf{y}=\mathbf{y}_{\alpha \alpha}$, it amounts to

$$
\mathbf{R}=\operatorname{diag}(2.1 \mathrm{e}-5,2.1 \mathrm{e}-5,2.6 \mathrm{e}-7,0.04,0.04) .
$$

For the other sensor sets, the speed measurement noise covariance value is set to $0.011(\mathrm{rad} / \mathrm{s})^{2}$.

The mechanical variables $\hat{\mathbf{x}}_{m}$ in the initial state vector $\mathbf{x}_{0}$ are set to the respective measured values of $\omega_{M}$ and $\theta_{M}$ at $t=t_{0}$. The electrical states $\hat{\mathbf{x}}_{e l}$ and the unknown load torque $\hat{T}_{L}$ are initialized to zero.

The initial state covariance matrix $\mathbf{P}_{0}$ is assumed diagonal. The elements corresponding to $\hat{\mathbf{x}}_{e l}$ match those of $\mathbf{Q}$ and the elements corresponding to $\hat{\mathbf{x}}_{m}$ match those of $\mathbf{R}$. The element corresponding to the load torque $\hat{T}_{L}$ is set to $1 \mathrm{e}-6 \mathrm{Nm}^{2}$.

\section{APPENDIX B StATE UPDATE COVARIANCES}

All values of $\mathbf{Q}$, except for the line and column of the unknown input, are determined according to the below procedure, based loosely on existing Monte-Carlo approaches [33], [34].

1) A nominal trajectory $\mathbf{x}_{n o m}(t)$ in the non-augmented state space is generated, based on the parameter values $\mathbf{p}_{\text {nom }}$ used implicitely in (22). In this work, $\mathbf{x}_{\text {nom }} \in \mathbb{R}^{n \times N}$ is obtained for $N$ time samples using a prior EKF implemented with a manually tuned diagonal matrix $\mathbf{Q}^{7}$.

2) Each of the $n_{p}$ model parameter uncertainties is quantified by its covariance value ${ }^{8}$. The result is a diagonal covariance matrix $\mathbf{Q}_{p}$. A set of sigma points $\chi_{i}^{p}$ is determined in analogy to (25). These are centered around $\mathbf{p}_{\text {nom }}$ and spreaded in proportion to $\sqrt{\mathbf{Q}_{p}}$.

3) For each sigma point $\chi_{i}^{p}, N$ model evaluations are done: $\mathbf{x}_{i}\left(t_{k}\right)=\mathbf{f}_{d, i}\left(\mathbf{x}_{n o m}\left(t_{k-1}\right), \mathbf{u}_{k}\right), \forall k \in[1, N]$. The deviations $\tilde{\mathbf{x}}_{i}(t)=\mathbf{x}_{i}(t)-\mathbf{x}_{\text {nom }}(t)$ are then computed.

4) Similarly to (28) and with the same weighting scheme, all trajectory deviations $\tilde{\mathbf{x}}_{i}$ are processed into the (now time-dependent) positive definite matrix: $\mathbf{Q}_{t}(t)=\Sigma_{i}\left(W_{i} \tilde{\mathbf{x}}_{i}^{T} \tilde{\mathbf{x}}_{i}\right)$. Linear time-averaging ${ }^{9}$ of $\mathbf{Q}_{t}$ yields the final result, i.e. the symmetric matrix $\mathbf{Q}_{f}$.

The informative value of $f_{u}$ is lower than that of $\mathbf{f}$. By lack of more precise knowledge, $Q_{u u}$ is assigned a value of either $\max \left(\mathbf{Q}_{f} \cdot\left[1 / n_{p}, \cdots, 1 / n_{p}\right]^{T}\right)$, denoted as low $Q_{u u}$, or this

\footnotetext{
${ }^{7}$ One could also obtain $\mathbf{x}_{n o m}(t)$ from pure simulation, choosing the model inputs to approximately cover the state space region of interest.

${ }^{8}$ In this work, all electrical model parameter uncertainties result directly from the model identification and all mechanical parameter uncertainties have been user-specified to a realistic value. One could also limit $\mathbf{Q}_{p}$ to a subset that contains only the most relevant parameters with a high uncertainty.

${ }^{9}$ Other averaging methods could be used to guarantee positivedefiniteness, but this has appeared unnecessary.
}

value multiplied by 1000 (high $Q_{u u}$ ). It is reminded that estimator results are insensitive to this value when the proposed sensor set $\mathbf{y}_{\alpha \alpha}$ is used. After verifying that estimator results are virtually unaffected by omitting the low off-diagonal values in $\mathbf{Q}_{f}$, only its diagonal is retained.

The above procedure has been carried out for four data sets, each with a duration of 4 seconds ( $N=16000$ samples). Although the operating trajectories differ significantly between these data sets, the four corresponding matrices $\mathbf{Q}_{f}$, hence also the resulting covariance matrix $\mathbf{Q}$ and the estimator results have all been verified to be practically indistinguishable.

This automated procedure is prone to underestimating uncertainties, because only parametric modeling errors are considered. However, the authors have found it to yield more accurate estimator results than those formerly obtained with extensive manual tuning.

\section{REFERENCES}

[1] N. Perišić, B. J. Pedersen, and P. H. Kirkegaard, "Gearbox fatigue load estimation for condition monitoring of wind turbines," in Proceedings of the International Conference on Noise and Vibration Engineering ISMA 2012, pp. 4391-4402, Leuven, 2012.

[2] N. Perišić, P. H. Kirkegaard, and B. J. Pedersen, "Cost-effective shaft torque observer for condition monitoring of wind turbines," Wind Energy, vol. 18, no. 1, pp. 1-19, 2015. [Online]. Available: https://doi.org/10.1002/we.1678

[3] S. H. Kia, H. Henao, and G.-A. Capolino, "Torsional vibration monitoring using induction machine electromagnetic torque estimation," in 34th Annual Conference of IEEE Industrial Electronics, pp. 3120-3125. Orlando: IEEE, 2008.

[4] S. H. Kia, H. Henao, and G.-A. Capolino, "Torsional vibration effects on induction machine current and torque signatures in gearboxbased electromechanical system," IEEE Transactions On Industrial Electronics, vol. 56, no. 11, pp. 4689-4699, 2009. [Online]. Available: https://doi.org/10.1109/TIE.2009.2026772

[5] S. H. Kia, H. Henao, and G.-A. Capolino, "Torsional vibration assessment using induction machine electromagnetic torque estimation," IEEE Transactions on Industrial Electronics, vol. 57, no. 1, pp. 209-219, 2010. [Online]. Available: https://doi.org/10.1109/TIE.2009.2034181

[6] H. Henao, S. H. Kia, and G.-A. Capolino, "Torsional-vibration assessment and gear-fault diagnosis in railway traction system," IEEE Transactions on Industrial Electronics, vol. 58, no. 5, pp. 1707-1717, 2011. [Online]. Available: https://doi.org/10.1109/TIE.2011.2106094

[7] J. Guzinski, M. Diguet, Z. Krzeminski, A. Lewicki, and A. Haithem, "Application of speed and load torque observers in high speed train drive for diagnostic purposes," IEEE Transactions On Industrial Electronics, vol. 56, no. 1, pp. 248-256, 2009. [Online]. Available: https://doi.org/10.1109/TIE.2008.928103

[8] A. Radke and G. Zhiqiang, "A survey of state and disturbance observers for practitioners," in Proceedings of the 2006 American Control Conference, pp. 5183-5186. Minneapolis: IEEE, 2006.

[9] R. E. Kalman, "A new approach to linear filtering and prediction problems," Transactions of the ASME-Journal of basic Engineering, vol. 82, no. 1, pp. 35-45, 1960.

[10] D. Simon, Optimal State Estimation. Hoboken: John Wiley \& Sons, 2006.

[11] S. Gillijns and B. De Moor, "Unbiased minimum-variance input and state estimation for linear discrete-time systems," Automatica, vol. 43, no. 1, pp. 111-116, 2007. [Online]. Available: https: //doi.org/10.1016/j.automatica.2006.08.002

[12] S. Gillijns and B. De Moor, "Unbiased minimum-variance input and state estimation for linear discrete-time systems with direct feedthrough," Automatica, vol. 43, no. 5, pp. 934-937, 2007. [Online]. Available: https://doi.org/10.1016/j.automatica.2006.11.016

[13] S. Julier, J. Uhlmann, and H. F. Durrant-Whyte, "A new method for the nonlinear transformation of means and covariances in filters and estimators," IEEE Transactions on Automatic Control, vol. 45, no. 3, pp. 477-482, 2000. [Online]. Available: https://doi.org/10.1109/9.847726

[14] S. Julier and J. Uhlmann, "Unscented Filtering and Nonlinear Estimation,” Proc. IEEE, vol. 92, no. 3, pp. 401-422, 2004. [Online]. Available: https://doi.org/10.1109/JPROC.2003.823141 
[15] R. van der Merwe, E. A. Wan, and S. J. Julier, "Sigma-Point Kalman Filters for Nonlinear Estimation and Sensor-Fusion," in AIAA Guidance, Navigation, and Control Conference and Exhibit, pp. 1-30. Providence: American Institute of Aeronautics and Astronautics, 2004.

[16] S. Jafarzadeh, C. Lascu, and M. S. Fadali, "State Estimation of Induction Motor Drives Using the Unscented Kalman Filter," IEEE Transactions On Industrial Electronics, vol. 59, no. 11, pp. 4207-4216, 2012. [Online]. Available: http://doi.org/10.1109/TIE.2011.2174533

[17] M. Anguelova, "Nonlinear observability and identifiability : General theory and a case study of a kinetic model for s. cerevisiae," Ph.D. dissertation, Chalmers University of technology and Götheborg University, 2004.

[18] B. T. Hinson, "Observability-based guidance and sensor placement," $\mathrm{Ph} . \mathrm{D}$. dissertation, University of Washington, 2014

[19] R. Hermann and A. J. Krener, "Nonlinear controllability and observability," IEEE Transactions on Automatic Control, vol. 60, no. 5, pp. 728-740, 1977.

[20] P. Vaclavek, P. Blaha, and I. Herman, "AC Drive Observability Analysis," IEEE Transactions on Industrial Electronics, vol. 60, no. 8 pp. 3047-3059, 2013. [Online]. Available: https://doi.org/10.1109/TIE. 2012.2203775

[21] M. Koteich, A. Maloum, G. Duc, and G. Sandou, "Discussion on "AC drive observability analysis'," IEEE Transactions on Industrial Electronics, vol. 62, no. 11, p. 7224, 2015. [Online]. Available: https://doi.org/10.1109/TIE.2015.2438777

[22] B. Forrier, R. Boonen, and W. Desmet, "Development of a novel test setup for validation of model-based virtual sensing on mechatronic drivetrains," in International Conference on Powertrain Modelling and Control, Loughborough, Sep.7-9 2016.

[23] "Lms imagine.lab amesim," www.plm.automation.siemens.com/en_us/ products/lms/imagine-lab/amesim, visited on Oct. 20, 2016.

[24] D. Karnopp, D. Margolis, and R. Rosenberg, System Dynamics: Modeling, Simulation, and Control of Mechatronic Systems, 2nd ed. Hoboken: John Wiley \& Sons, 2006.

[25] G. R. Slemon, "Modelling of induction machines for electric drives," IEEE Transactions on Industry Applications, vol. 25, no. 6, pp. 1126-1130, 1989. [Online]. Available: https://doi.org/10.1109/28.44251

[26] P. C. Krause, O. Wasynczuk, and S. D. Sudhoff, Analysis of Electric Machinery and Drive Systems, 2nd ed. New York: John Wiley \& Sons, 2002.

[27] H. C. Seherr-Thoss, F. Schmelz, and E. Aucktor, Universal Joints and Driveshafts: Analysis, Design, Applications, 2nd ed. Heidelberg: Springer-Verlag, 2006

[28] H. Trentelman, A. A. Stoorvogel, and M. Hautus, Control theory for Linear Systems, 2nd ed. London: Springer-Verlag, 2001.

[29] F. Naets, J. Cuadrado, and W. Desmet, "Stable force identification in structural dynamics using kalman filtering and dummy-measurements," Mechanical Systems and Signal Processing, vol. 50, pp. 235-248, 2015. [Online]. Available: http://dx.doi.org/10.1016/j.ymssp.2014.05.042

[30] R. F. Stengel, Optimal Control and Estimation. New York: Dover Publications, Inc., 1994

[31] T. Lefebvre, H. Bruyninckx, and J. D. Schutter, "Kalman filters for non-linear systems: a comparison of performance," International Journal of Control, vol. 77, no. 7, pp. 639-653, 2004. [Online]. Available: http://doi.org/10.1080/00207170410001704998

[32] S. Jafarzadeh, C. Lascu, and M. S. Fadali, "Square root unscented kalman filters for state estimation of induction motor drives," IEEE Transactions on Industry Applications, vol. 49, no. 1, pp. 92-99, 2013. [Online]. Available: http://doi.org/10.1109/TIA.2012.2229251

[33] J. Valappil and C. Georgakis, "A systematic tuning approach for the use of extended kalman filters in batch processes," in Proceedings of the American Control Conference (1999 AACC), pp. 1143-1147, San Diego, 1999.

[34] W. Rottiers, B. Forrier, M. Sarrazin, H. Van Der Auweraer, and W. Desmet, "Mechatronic powertrain virtual sensor: system-level model and sensor selection," in ICEDyn 2017 - International Conference on Structural Engineering Dynamics, Ericeira, 2017.

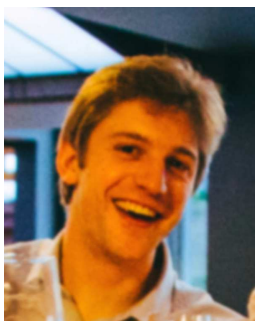

Bart Forrier was born in Halle, Belgium in 1989. $\mathrm{He}$ received the M.Sc. degree in mechanical engineering from KU Leuven, Belgium in 2011.

After graduation, he joined the Test Division of LMS International in Haasrode, Belgium. Since 2013, he has been with the Noise \& Vibration research group at $\mathrm{KU}$ Leuven, where he is currently pursuing a Ph.D. degree.

M.Sc. Forrier is an active member of Flanders Make, the Strategic Research Centre for the Manufacturing Industry. His main research interests are in noise \& vibration engineering and model based virtual sensing. His related efforts focus on mechatronic powertrain applications in industrial machines, vehicles and wind turbines.

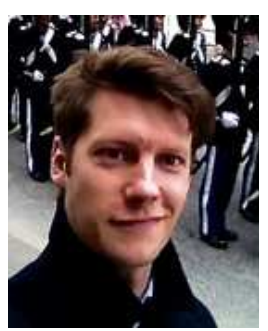

Frank Naets was born in Leuven, Belgium in 1986. He received the M.Sc. degree (2009) and a Ph.D. degree (2013) in mechanical engineering from KU Leuven, Belgium.

Since 2009, he has been with the Noise \& Vibration research group at KU Leuven, where he is currently working as a post-doctoral researcher (FWO Fellow).

Dr. Naets is an active member of Flanders Make, the Strategic Research Centre for the Manufacturing Industry. His main research interests are in the fields of dynamic mechanical modelling, model order reduction and model based virtual sensing. His related efforts focus on dynamic mechatronic systems and vehicle dynamics.

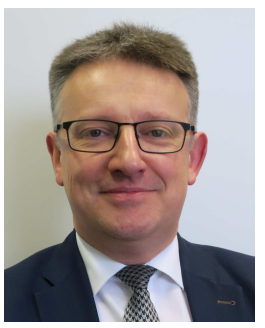

Wim Desmet was born in Waregem, Belgium in 1969. He obtained the M.Sc. degree (1992) and a Ph.D. degree (1998) in mechanical engineering from $\mathrm{KU}$ Leuven, Belgium.

$\mathrm{He}$ is a Full Professor in dynamics and mechatronics at the KU Leuven Department of Mechanical Engineering, where he is currently the head of the Noise \& Vibration Research group. His research interests include virtual prototyping techniques in vibro-acoustics, structural dynamics, aero-acoustics and (flexible) multibody dynamics, experimental approaches for advanced dynamic testing and identification, model based virtual sensing, mechatronic system simulation, vehicle mechatronics, noise control engineering, dynamics of lightweight systems, and structural health monitoring. He has published over 220 papers in international peerreviewed journals.

Prof. Desmet is the Director of the Mechatronics \& Design Methods Department of Flanders Make, the Strategic Research Centre for the Manufacturing Industry. $\mathrm{He}$ is the Chairman of the KU Leuven Industrial Research Fund. $\mathrm{He}$ is the General Chairman of the biennial ISMA-Noise and Vibration Engineering Conference in Leuven, Belgium. He is member of the General Assembly of EARPA-European Automotive Research Partners Association, and he is the representative for KU Leuven in EGVIA-European Green Vehicles Initiative Association. 